\title{
The Relationship between Classroom Environment and EFL Learners' Academic Self-Efficacy
}

\author{
Masoumeh Naghsh Daemi ${ }^{1}$, Abdorreza Tahriri ${ }^{2 *}$, Amir Mahdavi Zafarghandi ${ }^{3}$ \\ ${ }^{1} M A$ in TEFL, Department of English Language and Literature, University of Guilan, Iran, \\ ${ }^{2}$ Assistant professor of Applied Linguistics, Department of English Language and Literature, University of Guilan, Iran
}

Corresponding author: Abdorreza Tahriri, E-mail: atahriri@gmail.com

\section{ARTICLE INFO \\ Article history \\ Received: August 16, 2017 \\ Accepted: September 27, 2017 \\ Published: October 31, 2017 \\ Volume: 5 Issue: 4}

Conflicts of interest: None

Funding: None

\begin{abstract}
The present study sought to examine the relationship between classroom environment and English as a Foreign Language (EFL) learners' academic self-efficacy. To this end, a sample of 200 advanced EFL learners (146 females and 54 males) completed the What is Happening In This Class? (WIHIC) which consists of seven scales including Student Cohesiveness, Teacher Support, Involvement, Investigation, Task Orientation, Cooperation, and Equity that help to measure classroom learning environment. The Self-Efficacy for Learning Form (SELF-A) was also administered to gauge the participants' academic self-efficacy. In order to analyze the data, Spearman rank-order correlation was run. The results revealed that there was a significant relationship between EFL learners' classroom environment and their self-efficacy $(\mathrm{rho}=.438)$. The findings reflected that the highest relationship was between task orientation and self-efficacy $($ rho $=.433)$ followed by the relationship between student cohesiveness and self-efficacy (rho $=.353$ ). However, the lowest relationship was found between cooperation and self-efficacy (rho $=.199$ ). Overall, the results highlight the relationship between classroom environment and academic self-efficacy.
\end{abstract}

Key words: Academic Self-Efficacy, Classroom Environment, Learners, EFL

\section{INTRODUCTION}

Learners with similar aptitude and capacities may have a different performance in learning a language. To shed light on this issue, researchers have tried to consider some affective factors, such as the learners' beliefs in themselves in performing a task (Bandura, 1997), their perceptions of the task (Williams \& Burden, 1997) and some other individual differences such as learning strategies (Cohen, 1998) and motivation (Dörnyei, 2005).

In 1986, Bandura stated that "many students have difficulty in school not because they are incapable of performing successfully, but because they are incapable of believing that they can perform successfully" (p. 390), or they do not have enough self-efficacy. In education, self-efficacy is a critical contributing element to students' development, because self-efficacy might influence the decisions students make and the strategies they seek after. In fact, academic self-efficacy is rooted in self-efficacy theory.

Self-efficacy theory was begun from social cognitive theory by Bandura and is characterized as people's beliefs about their capacities to perform an activity. It is identified with one's view of his/her capacity to achieve an objective, and it is known as a critical concept in positive psychology. Self-efficacy is considered as the most essential precondition for changes that occur in one's behavior (Bandura, 1977). We should keep in mind that self-efficacy beliefs are multi-di- mensional (Zimmerman \& Kitsantas, 1997). The present research concentrated on the area of academic self-efficacy which relates to students' motivation and it refers to students' confidence in their capability to learn or do particular tasks (Bandura, 1986, 1997) or according to Zimmerman (1995) it is personal judgement of someone's abilities to sort out and execute courses of action to accomplish assigned types of instructive performances.

Academic self-efficacy is perceived as a constituent of student motivation and is clarified as the beliefs that students possess in their capability to learn or conduct specific tasks (Bandura, 1986, 1997). Those students who have high self-efficacy go through demanding tasks regularly and have tendency to gain higher than students with low self-efficacy (Pajares, 1996). On the other hand, those students who have low self-efficacy stop continuing their attempts in the case of failure which reduces their success and sense of self-efficacy.

The most consistent finding regarding EFL context is that EFL learners' self-efficacy influences their performance in various domains of language (Lane, Lane, \& Kyprianou, 2004; Moghari, Lavasani, Bagherian, Afshari 2011; Rahimpour \& Nariman-jahan 2010, as cited in Raoofi, Tan, \& Chen, 2012).

Various factors can be influential in forming one's behaviors and attitudes, such as his/her environment, interactions 
with other people especially peers, receiving feedback, and his/her own experience (Battistich, Solomon, Kim, Watson, \& Schaps, 1995). Classroom environment refers to the personal, educational, social, and psychological context of a classroo. The teacher has influential roles; the impacts of students themselves cannot be ignored, and the quality of classroom also is effective in learners' feeling and learning (Schmuck \& Schmuck, 1974). Environment and individuals are in direct relation with each other and it is not possible to consider them separately. The quality of classroom environment influences the students' self-image, confidence, learning, and attitude towards the subject. In particular, classroom environment includes a wide scope of educational concepts like the physical setting, the psychological environment, and various instructional components.

In spite of the existence of a variety of past studies regarding the relationship between the mathematics classroom environment and student learning, in second language learning, few studies have been conducted with the purpose of finding factors influencing the development of self-efficacy beliefs. In fact, classroom environment has been researched extensively, as well as self-efficacy, but very few studies have looked at the relationship between the two (Spinner \& Fraser, 2005; Wang, 2012).

Considering the influential role that beliefs and thoughts have, it is of importance to do further studies on learners' self-efficacy and how to boost it in educational settings including institutes, schools, and universities. Therefore, there is a need to study the relationship between classroom environment and EFL learners' academic self-efficacy.

\section{LITERATURE REVIEW}

Academic self-efficacy was proved to be an important factor in academic performance in the study conducted by Chemers, Hu, and Garcia (2001). In this study, first-year college students answered Academic Self-Efficacy Scale. It was revealed that those who had high academic self-efficacy also had higher grade point averages (GPAs). Studies in the realm of learning environments began with the endeavors of Lewin and Murray in the 1930s and advanced through with Walberg and Moos in the 1960s who paved the way for additional research all over the world (Madu, 2010).

Dorman (2001) employed 1055 mathematics secondary school students in Australia and asked them to complete two instruments (What Is Happening In This Classroom? and the Constructivist Learning Environment Survey). The purpose was to examine the relationship between classroom psychosocial environment and academic efficacy. The analyses of the obtained data revealed a positive association between the environment and academic efficacy with the dimensions of WIHIC scale having greater relationship rather than the other scale.

A similar study was conducted in Canada too. Ferguson and Dorman (2001) examined the relationship between classroom environment and academic efficacy among high school mathematics students. Nine hundred and fifty-one students (490 male and 461 female) took part in the study and completed the questionnaires: WIHIC, CLES and a 7-item scale utilizing items created by Midgley et al. (1997). The gathered data were analyzed through simple and multiple correlation. The simple correlations between the classroom environment scales and Academic Efficacy were measurably noteworthy. The correlations were from .10 for Shared Control with Academic Efficacy to .46 for Task Orientation with Academic Efficacy. Multiple correlation analyses showed that the 10 classroom environment scales represented $31.4 \%$ of variance in Academic Efficacy. Standardized regression coefficients for these examinations proposed that Task Orientation had the most intense impact on academic efficacy $(\beta=0.35)$. The conclusion of this study showed that various critical classroom environment dimensions are related essentially with academic efficacy. For instance, enhanced levels of Involvement, Investigation, and Task Orientation were related with higher levels of Academic Efficacy.

The analysis of the data from Anderson's (2004) study showed a positive relationship between academic self-efficacy and classroom environment, and showed that enhancement of cohesiveness, satisfaction, and goal direction from classrooms environment can increase academic self-efficacy and achievement.

Dorman and Adams (2004) studied 2651 mathematics students about 8 to 12 years of age from nine Australian and sixteen British secondary schools. The purpose was to find the relationships between classroom environment and students' academic efficacy and also to specify the strongest effective factor of classroom environment on their academic efficacy in mathematics. The participants gave the information about their capability to reach their academic goals and their view of the classroom environment by answering two different questionnaires. Academic efficacy questionnaires included seven items and the classroom environment questionnaire consisted of 60 questions which was the combination of two separate instruments: The What Is Happening In This Classroom? (WIHIC) with 42 items and the Constructivist Learning Environment Survey (CLES) with 18 items. Finally, they found that equity, task orientation and teacher support were in connection with academic efficacy in mathematics.

The objectives of Dorman, Waldrip, and Fisher's (2006) research were to recognize classroom environment and view of assessment dimensions that foresee academic efficacy and attitude to science. Four hundred and forty-nine students in secondary schools in Queensland participated in the study. They were surveyed in science classrooms and it was significant to the current study because all variables under examination were subject-specific. Finally, it was found that classroom environment and learners' view of assessment were strong positive predictors of academic efficacy and attitude to science.

McMahon, Wernsman, and Rose (2009) carried out a study in order to explore the relationship between classroom environment and academic self-efficacy among fifth and sixth grade students from California. The findings confirmed the relationship and revealed that academic self-efficacy could be influenced by classroom environment.

In a study carried out in Spain by Valle et al. (2009), the 
researchers sought to find the relationship between self-efficacy for performance and learning and effort regulation of a group of university students. The results showed that the students who had higher self-efficacy put more effort into their academic studies.

Chinese and Malay students' academic efficacy was also proved to be affected by their learning environment. Lim (2013) employed 441 students from Singapore and utilized the WIHIC, the Test of Science Related Attitudes (TOSRA) and the Morgan-Jinks Student Efficacy Scale (MJSES) as the instruments of the study. Involvement and Equity were considered as the significant predictors of efficacy.

Another study was carried out in the USA and Hong Kong. In Hanke's (2013) study 1,309 seventh and eighth mathematics students participated and completed the WIHIC questionnaire, Attitudes towards Mathematical Inquiry and Enjoyment of Mathematics from the Test of Mathematics-Related Attitudes (TOMRA), and Morgan-Jinks Student Efficacy Scale (MJSES). The results showed significant and positive associations between learning environment and students' attitudes towards mathematics academic self-efficacy. Involvement was suggested to have the strongest effect on academic self-efficacy.

Another study looking for the relationship between classroom environment and students' self-efficacy in mathematics was conducted in a school in North Texas with grades 4 through 12 students. Croissant (2014) utilized two instruments in the study that were My Classroom Inventory (MCI) and Patterns of Adaptive Learning Survey (PALS) that students completed in their regular school day. It was found that high self-efficacy in mathematics would increase as cohesion and satisfaction increase, and friction and difficulty decrease.

Zedan and Bitar (2014) also confirmed the positive relationship between classroom environment and students' self-efficacy. This study was conducted with 900 high school students in Israel. The participants were asked to complete the classroom climate questionnaire in the mathematics lesson, and mathematical self-efficacy questionnaire. The regression analysis revealed that mathematical self-efficacy was effective in predicting achievements in mathematics.

The focus of the present study was to investigate the relationship between the dimensions of classroom environment and EFL learners' academic self-efficacy. As such, the present study sought to answer the following research question and test the relevant null hypothesis:

RQ. Is there any statistically significant relationship between classroom environment and EFL learners' academic self-efficacy?

$\mathrm{H}_{0}$. There is no statistically significant relationship between classroom environment and EFL learners' academic self-efficacy.

\section{METHOD}

\section{Participants}

The participants of the study were 200 advanced EFL learners studying English in Iran Language Institutes (ILI) of Guilan province, Iran. The participants were selected based on convenience sampling. There were both male $(n=54)$ and female $(n=146)$ participants in this study. Overall, 73\% of them were female and $27 \%$ of them were male learners.

\section{Instruments}

In order to collect information from the participating students, two questionnaires were utilized in this study:

\section{What is Happening In This Class? (WIHIC)}

This questionnaire consists of seven scales and 56 items. The scales are Student Cohesiveness, Teacher Support, Involvement, Investigation, Task Orientation, Cooperation, and Equity that help to measure classroom learning environment. This instrument was developed by Fraser, Fisher, and McRobbie (1996, as cited in Madu, 2010) and has been used and cross-validated in different researches including Aldridge, Fraser, and Huang (1999), Dorman (2003), Margianti, Fraser, and Aldridge (2004), Chionh and Fraser (2009) among others (cited in Madu, 2010). Therefore, the validity and reliability of this scale have been confirmed for investigating students' perceptions toward their learning environment. In the current study, the reliability index estimate for the "WIHIC Questionnaire" was also computed $(\alpha=.906)$.

\section{Self-Efficacy for Learning Form (SELF-A)}

The developers of SELF were Zimmerman and Kitsantas (2005). At first, the SELF with 57 items was organized to assess students' perceived self-efficacy including different forms of academic work like reading, note taking, test taking, writing and studying. Then, it was converted to the form of abridged SELF with 19 items. The purpose behind this conversion was to reach the goals in a more efficient and less time-consuming process. The SELF-A was effective in assessing students' academic self-efficacy beliefs with the focus on studying, test-preparation and note-taking. The respondents were supposed to specify their level of confidence in conducting a particular activity by mentioning the percentage next to each statement. Based on the guidelines provided above the statements, the answers ranged from $0 \%$ (Definitely cannot do it) to $100 \%$ (Definitely can do it). In the study of Zimmerman and Kitsantas (2007) with the purpose of determining the validity of SELF scores, students' scores were reported to have a unitary factor structure and was highly reliable (Both SELF and SELF-A, even with the superiority of SELF-A in prediction of most validity measures). In the present study the value of Cronbach's Alpha was also computed $(\alpha=.777)$.

\section{Procedure}

In order to make sure that the participants understand the items of the questionnaire, the English form of both questionnaires (WIHIC and SELF-A) were translated into Persian and back translated to English by two language experts to ensure the validity of translation. Next, a pilot study was conducted to ensure the appropriateness and reliability of the 
instruments. After arrangements with the authorities of ILI, the translated versions of the questionnaires were distributed among students either before or after the regular class time. Prior to the survey, the students were provided with a verbal explanation of the purpose of the study and the instruction for completing the questionnaire. The respondents were asked to specify their opinions either by selecting from the range in WIHIC from "almost never" to "almost always", or by writing the percentages that suited them next to each statements in SELF-A. They also entered some demographic information in the survey including gender, age, and period of studying English, but in order to preserve their anonymity, they were not supposed to write their names. In addition, they were given the confidence that their information would be used for research work only. After the data were gathered, they were analyzed using SPSS (version 23).

\section{Data Analysis}

In order to analyze the data, Spearman rank-order correlation was run to examine the relationship between classroom environment and EFL learners' academic self-efficacy. Before examining the possible relationship between the two variables, descriptive statistics was, first and foremost, computed for the EFL learners' academic self-efficacy and the seven subscales of the classroom environment questionnaire. Descriptive statistics including measures of central tendency, measures of dispersion, and measures of distribution were computed. To evaluate the extent of the shared variance between the two variables, the coefficient of determination was calculated. The rho value was squared and then changed to percentage of variance; afterwards, it was multiplied by 100 .

\section{RESULTS}

Before examining the relationship between the two variables, first, descriptive statistics was computed for the EFL learners' academic self-efficacy and the seven subscales of the classroom environment questionnaire. For the categorical ordinal data obtained for the seven components of the classroom environment including student cohesiveness, teacher support, involvement, investigation, task orienta- tion, cooperation, and equity, the descriptive data comprised the mean rank, median, and range. See Table 1.

With respect to the mean rank for the total sample, the highest mean rank was reported for the equity component (mean rank $=4.37$ ) closely followed by the task orientation (mean rank $=4.09$ ). In contrast, the lowest mean rank was reported for the investigation part (mean rank $=2.84$ ).

For the EFL leaners who reported low academic self-efficacy, the highest mean rank was reported for the equity component (mean rank $=4.21$ ) closely followed by the task orientation (mean rank $=3.62$ ). In contrast, the lowest mean rank was reported for the investigation section (mean rank $=2.68)$.

When it comes to the EFL leaners who reported high academic self-efficacy, the highest mean rank was reported for the equity component (mean rank $=4.39$ ) closely followed by the task orientation (mean rank $=4.17$ ). In contrast, the lowest mean rank was reported for the investigation section (mean rank $=2.87$ ).

In general, EFL learners with high levels of academic self-efficacy reported higher mean ranks for all the seven categories of classroom environment compared to the EFL learners with low levels of academic self-efficacy. Descriptive statistics including measures of central tendency, measures of dispersion, and measures of distribution were computed for the results of the self-efficacy questionnaire, too.

As it is given in Table 2, the total mean for the EFL learners' academic self-efficacy amounted to be $63.29(S D=13.83)$. The minimum and maximum degrees of academic self- efficacy were 25.79 and 97.89 , respectively.

Following the descriptive statistics, to provide an answer to the research question and to examine the relationship between EFL learners' ratings for the classroom environment (categorical ordinal data) and their self-efficacy (interval data), Spearman rank-order correlation test was run. The results are presented in Table 3:

Table 3 showed the results of the Spearman rank-order correlation test that computed the extent of the correlation between EFL learners' ratings for the classroom environment and their self-perceived academic self-efficacy. Table 4 presents the guideline used to interpret the rho value found in the present study.

Table 1. Descriptive statistics for the subscales of the classroom environment questionnaire with respect to low and high levels of self-efficacy

\begin{tabular}{|c|c|c|c|c|c|c|c|c|c|}
\hline & \multicolumn{9}{|c|}{ Self-efficacy } \\
\hline & \multicolumn{3}{|c|}{ Mean Rank } & \multicolumn{3}{|c|}{ Median } & \multicolumn{3}{|c|}{ Range } \\
\hline & Low & High & Total & Low & High & Total & Low & High & Total \\
\hline Student cohesiveness & 3.42 & 3.86 & 3.80 & 3.62 & 4.00 & 3.87 & 3.13 & 2.63 & 3.13 \\
\hline Teacher support & 2.97 & 3.33 & 3.27 & 3.00 & 3.37 & 3.31 & 2.88 & 3.50 & 3.63 \\
\hline Involvement & 2.92 & 3.35 & 3.29 & 3.12 & 3.25 & 3.25 & 2.38 & 3.50 & 3.50 \\
\hline Investigation & 2.68 & 2.87 & 2.84 & 2.75 & 2.87 & 2.87 & 3.88 & 3.75 & 3.88 \\
\hline Task orientation & 3.62 & 4.17 & 4.09 & 3.75 & 4.25 & 4.25 & 2.63 & 4.13 & 4.13 \\
\hline Cooperation & 3.38 & 3.70 & 3.66 & 3.37 & 3.75 & 3.62 & 3.38 & 3.75 & 3.75 \\
\hline Equity & 4.21 & 4.39 & 4.37 & 4.50 & 4.75 & 4.75 & 2.75 & 3.00 & 3.00 \\
\hline
\end{tabular}


Table 2. Descriptive statistics for the self-efficacy inventory

\begin{tabular}{lc}
\hline N & \\
Valid & 200 \\
Missing & 0 \\
Mean & 63.2982 \\
Median & 63.2895 \\
Mode & 63.16 \\
Standard deviation & 13.83169 \\
Variance & 191.316 \\
Skewness & -0.025 \\
Std. Error of Skewness & 0.172 \\
Kurtosis & 0.377 \\
Std. Error of Kurtosis & 0.342 \\
Range & 72.11 \\
Minimum & 25.79 \\
Maximum & 97.89 \\
Sum & 12659.63 \\
\hline
\end{tabular}

Table 3. Spearman correlation for the classroom environment and EFL learners' academic self-efficacy $(n=200)$

\begin{tabular}{|c|c|}
\hline & Self-efficacy \\
\hline \multicolumn{2}{|l|}{ Spearman's rho } \\
\hline \multicolumn{2}{|l|}{ Student cohesiveness } \\
\hline Correlation coefficient & $0.353 * *$ \\
\hline Sig. (2-tailed) & 0.000 \\
\hline \multicolumn{2}{|l|}{ Teacher support } \\
\hline Correlation coefficient & $0.266^{* *}$ \\
\hline Sig. (2-tailed) & 0.000 \\
\hline \multicolumn{2}{|l|}{ Involvement } \\
\hline Correlation coefficient & $0.349 * *$ \\
\hline Sig. (2-tailed) & 0.000 \\
\hline \multicolumn{2}{|l|}{ Investigation } \\
\hline Correlation coefficient & $0.264 * *$ \\
\hline Sig. (2-tailed) & 0.000 \\
\hline \multicolumn{2}{|l|}{ Task orientation } \\
\hline Correlation coefficient & $0.433 * *$ \\
\hline Sig. (2-tailed) & 0.000 \\
\hline \multicolumn{2}{|l|}{ Cooperation } \\
\hline Correlation coefficient & $0.199 * *$ \\
\hline Sig. (2-tailed) & 0.005 \\
\hline \multicolumn{2}{|l|}{ Equity } \\
\hline Correlation coefficient & $0.247 * *$ \\
\hline Sig. (2-tailed) & 0.000 \\
\hline \multicolumn{2}{|l|}{ Total } \\
\hline Correlation coefficient & $0.438 * *$ \\
\hline Sig. (2-tailed) & 0.000 \\
\hline
\end{tabular}

**Correlation is significant at the 0.01 level (2-tailed)
For the first subscale of the classroom environment questionnaire that was related to the student cohesiveness, there was a medium correlation between EFL learners' ratings for the student cohesiveness component and their reflected academic self-efficacy (rho $=.353$ ). The significance value was lower than. 01 indicating that the correlation was statically significant for the two variables ( $p$ $<.01)$. For the second subscale of the questionnaire that dealt with the teacher support, there was a small correlation between the learners' ratings for the teacher support component and their academic self-efficacy (rho $=.266$ ). The significance value was lower than. 01 implying that the correlation was statically significant for the two variables $(p<.01)$. For the third subscale that inspected the involvement, there was a medium correlation between the learners' ratings for the involvement component and their academic self-efficacy (rho $=.349$ ). The significance value was lower than (.01) suggesting that the correlation was statically significant for the two variables $(p<.01)$. For the fourth subscale that was related to the investigation, there was a small correlation between the learners' ratings for the investigation component and their academic self-efficacy (rho $=.264$ ). The significance value was lower than.01 displaying that the correlation was statically significant for the two variables $(p<.01)$. For the fifth subscale that was related to the task orientation, there was a medium correlation between the learners' ratings for the task orientation component and their academic self-efficacy $($ rho $=.433)$. The significance value was lower than. 01 showing that the correlation was statically significant for the two variables $(p<.01)$. For the sixth subscale that was related to the cooperation, there was a small correlation between the learners' ratings for the cooperation component and their academic self-efficacy (rho $=.199)$. The significance value was lower than. 01 resulting that the correlation was statically significant for the two variables $(p<.01)$. For the seventh subscale that was related to the equity, there was a small correlation between the learners' ratings for the equity component and their academic self-efficacy (rho =.247). The significance value was lower than. 01 resulting that the correlation was statically significant for the two variables $(p<.01)$.

In general, there was a medium correlation between the EFL learners' ratings for the total classroom environment and their reflected academic self-efficacy (rho =.438). The significance value was lower than. 01 indicating that the correlation was statically significant for the classroom environment and EFL learners' reflected academic self-efficacy $(p<.01)$.

Table 5 lists the seven components of the classroom environment from the highest to the lowest based on the relationship between the above-mentioned components and self-efficacy:

The findings reflected that the highest relationship was found between task orientation and self-efficacy $($ rho $=.433$ ) followed by the relationship between student cohesiveness and self-efficacy (rho =.353). However, the lowest one was found for the relationship between cooperation and self-effi$\operatorname{cacy}($ rho $=.199)$. 
Table 4. Cohen's (1988) guidelines for interpreting the results of the correlation coefficient

\begin{tabular}{lc}
\hline Interpretation & Correlation value \\
\hline Small correlation & 0.10 to 0.29 \\
Medium correlation & 0.30 to 0.49 \\
Large correlation & 0.50 to 1.0 \\
\hline
\end{tabular}

Table 5. Correlation between the subscales of the classroom environment and EFL learners' academic self-efficacy

\begin{tabular}{lcl}
\hline Components & Correlation index & Interpretation \\
\hline Task orientation & 0.433 & Medium \\
Student cohesiveness & 0.353 & Medium \\
Involvement & 0.349 & Medium \\
Teacher support & 0.266 & Small \\
Investigation & 0.264 & Small \\
Equity & 0.247 & Small \\
Cooperation & 0.199 & Small \\
\hline
\end{tabular}

To evaluate the extent of the shared variance between the two variables, the coefficient of determination was calculated. The (rho) value was squared and then changed to percentage of variance; afterwards, it was multiplied by 100 . In this study, the participants' ratings for the student cohesiveness and their self-efficacy correlated (rho =.353) share $12.46 \%$ of their variance. The participants' ratings for the teacher support and their self-efficacy correlated (rho $=.266$ ) share $7.07 \%$ of their variance. The participants' ratings for the involvement and their self-efficacy correlated (rho=.349) share $12.18 \%$ of their variance. The participants' ratings for the investigation and their self-efficacy correlated (rho $=.264$ ) share $6.96 \%$ of their variance. The participants' ratings for the task orientation and their self-efficacy correlated $($ rho $=.433)$ share $18.74 \%$ of their variance. The participants' ratings for the cooperation and their self-efficacy correlated (rho $=.199$ ) share $3.96 \%$ of their variance. The participants' ratings for the equity and their self-efficacy correlated (rho $=.247$ ) share $6.10 \%$ of their variance. Finally, the participants' ratings for the total classroom environment and their self-efficacy correlated (rho $=.438$ ) share $19.18 \%$ of their variance.

Therefore, there was a small overlap between the two variables. This implied that the EFL learners' ratings for the total classroom environment helped to explain simply $19.18 \%$ of the variance in their self-efficacy. In sum, the small positive correlation found between the two variables, (rho $=+.438$, $n=200, p<.01$ ) rejected the null hypothesis and suggested that there was a significant relationship between EFL learners' classroom environment and their self-efficacy.

As it is depicted in Figure 1, the participants' self-perceived academic self-efficacy moderately correlated with their classroom environment components. In other words, those who had higher ratings for the subscales of the classroom environment relatively reflected higher levels of self-efficacy and vice versa.

\section{DISCUSSION}

The aim of the present study was to investigate the relationship between classroom environment and academic self-efficacy of a group of EFL learners. The analysis of the findings proved the existence of such a relationship.

As a whole, EFL learners with high academic self-efficacy reported higher mean ranks for the seven components of the classroom environment compared to the EFL learners with low levels of academic self-efficacy. Moreover, the results of Spearman rank-order test revealed that there was a statistically significant relationship between EFL learners' self-perceived self-efficacy levels and the classroom environment. In addition, it was also found that the highest relationship was between task orientation and self-efficacy $($ rho $=.433)$ followed by the relationship between student cohesiveness and self-efficacy (rho =.353). However, the lowest relationship was between cooperation and self-efficacy (rho $=.199)$.

In line with the obtained results of the present study, Dorman's (2001) findings revealed a positive association between the environment (with the dimensions of WIHIC scale) and academic efficacy.

Dorman, Waldrip, and Fisher's (2006) study also confirmed the positive relationship between classroom environment and academic self-efficacy in science classes. The results of the present study are also in agreement with $\mathrm{Ca}$ nadian high school mathematics students' opinions toward their classroom environment. Ferguson and Dorman (2001) also found that task orientation had the most intense impact on the students' higher levels of academic efficacy.

Anderson (2004) also found a positive relationship between academic self-efficacy and classrooms environment. Besides, it was shown that by developing cohesiveness, satisfaction, and goal direction, students' academic self-efficacy and achievement could be increased. These results support the conclusions of the current study that high academic self-efficacy increases when cohesiveness increases. Moreover, it supports the social-cognitive theory too indicating that expectations can be determined by beliefs and while students experience a classroom in which cohesiveness and satisfaction are paid attention to and enhanced, they can have positive effects on their future behavior ( $\mathrm{Pa}-$ jares, 1996).

Similar to Dorman and Adams's (2004) study who found that equity, task orientation and teacher support were in connection with academic efficacy of Australian and British mathematics students, task orientation was considered as the most effective component on academic efficacy. Cohesiveness was also suggested to be one positive predictor factor on high math self-efficacy in Croissant's (2014) study, but this time in North Texas. The analysis of her study (just like the current study) was also not able to generalize low self-efficacy in mathematics as the result of the small effect size.

The conclusion of the current study is also supported by a cross-national study held in Hong Kong and USA. Hanke (2013) employed 1309 seventh and eighth mathematics students as the participants and found significant positive relationships between classroom learning environment and 


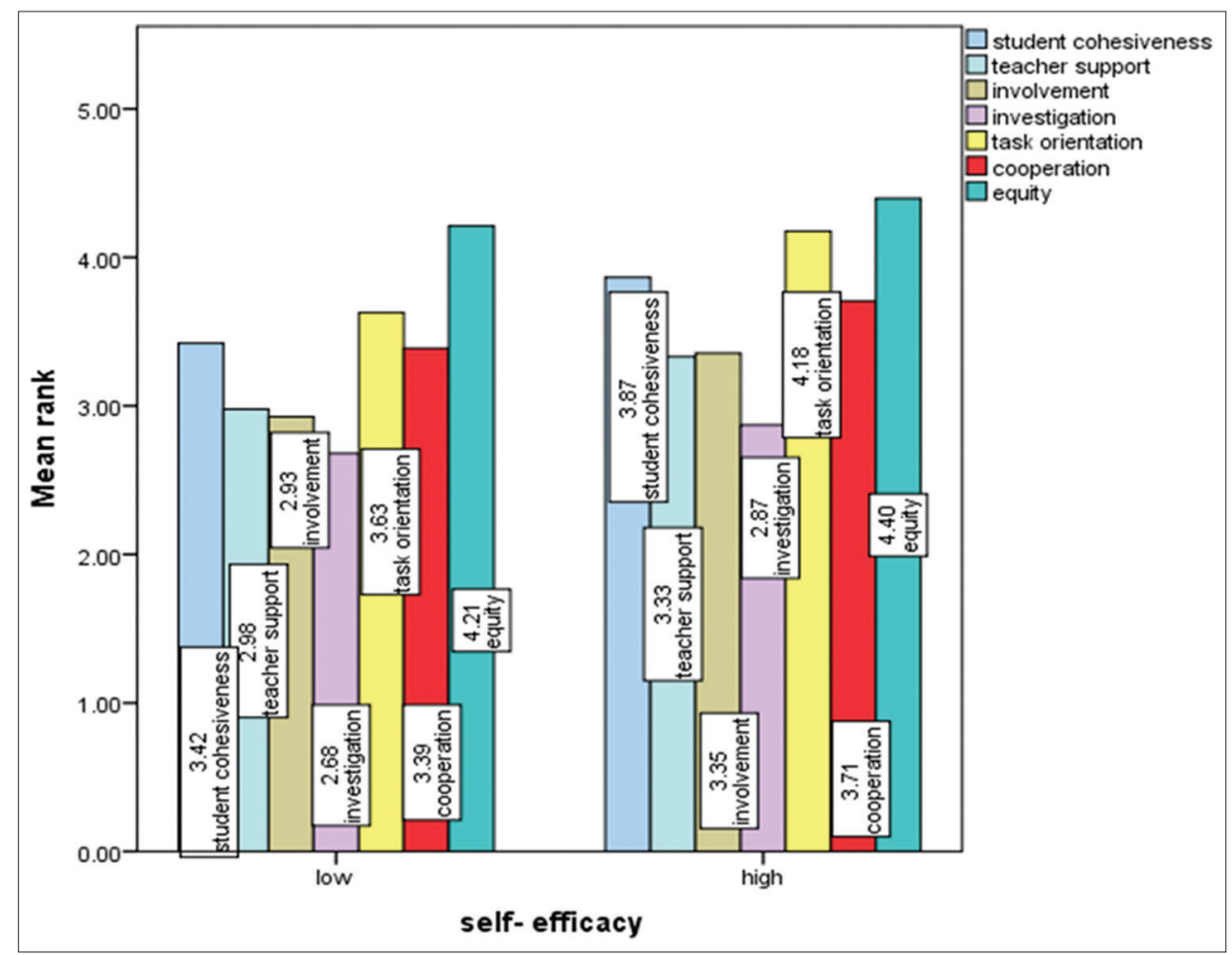

Figure 1. The Ratings for the Classroom Environment Subscales by Low and High Self-Efficacy EFL Learners

learners' academic self-efficacy. In Hanke's (2013) study, the significant predictor of academic self-efficacy in both countries was involvement and this determinant was shown to be stronger in Hong Kong than in the USA.

\section{CONCLUSION}

To find an answer to the research question, two questionnaires were piloted and then distributed among 200 advanced students at ILI. The purpose was to investigate the relationship between EFL learners' ratings for the classroom environment (categorical ordinal data) and their self-efficacy (interval data). The results of Spearman rank-order correlation revealed that there was a medium correlation between EFL learners' ratings for the total classroom environment and their reflected academic self-efficacy. The findings showed that the highest relationship was found between task orientation and self-efficacy followed by the relationship between student cohesiveness and self-efficacy. However, the lowest relationship was between cooperation and self-efficacy.

Policy makers and administrators can take into account the findings of this study, to enhance the academic self-efficacy of EFL learners. The findings provide them with new insights into the classroom environment and help them in making proper decisions to have the most fruitful classrooms. By considering the results of the current study, educators feel the need to create such an environment in their classroom that fosters high English self-efficacy and decreases low English self-efficacy. Teachers can inspect their students' views towards their classroom, and attempt to develop more beneficial dimensions. In addition, teachers and educators can use the results of this study to lead their classes to maximize the satisfactory aspects of their classroom environment, which can lead to higher academic self-efficacy in students. Being aware of the beneficial effects of classroom environment on the improvement of academic self-efficacy, students can consider and pay attention to those dimensions that they themselves can manipulate and have control over.

\section{REFERENCES}

Anderson, L. (2004). From chaos to community. Retrieved from https://www.tolerance.org/classroom-resources/ tolerance-lessons/from-chaos-to-community

Bandura, A. (1977). Self-efficacy: Toward a unifying theory of behavioral change. Psychological Review, 84(2), 191-215.

Bandura, A. (1986). Social foundations of thought and action: A social cognitive theory. Englewood Cliffs, NJ: Prentice Hall.

Bandura, A. (1997). Self-efficacy: The exercise of control. New York: W. H. Freeman and Company.

Battistich, V., Solomon, D., Kim, D., Watson, M., \& Schaps, E. (1995). Schools as communities, poverty levels of student populations, and students' attitudes, motives, and performance: A multilevel analysis. American Educational Research Journal, 32(3), 627-658. doi:10.3102/00028312032003627

Chemers, M. M., Hu, L. T., \& Garcia, B. F. (2001). Academic self-efficacy and first year college student performance and adjustment. Journal of Educational Psychology, 93(1), 55-64.

Cohen, A. D. (1998). Strategies for learning and using a second language. New York: Longman. 
Croissant, H. (2014). Classroom environment influence on student self-efficacy in mathematics. Texas A \& M University Commerce. Retrieved from http://gradworks. umi.com/36/20/3620807.html

Dorman, J. P. (2001). Associations between classroom environment and academic efficacy. Learning Environments Research, 4(3), 243-257.

Dorman, J., \& Adams, J. (2004). Associations between students' perceptions of classroom environment and academic efficacy in Australian and British secondary schools. Westminster Studies in Education, 27(1), 69-85.

Dorman, J., Waldrip, B., \& Fisher, D. L. (2006). Classroom environment, students' perceptions of assessment, academic efficacy and attitude to science: A LISREL analysis. In proceedings of the NARST 2006 Annual Meeting (pp. 1-32). National Association for Research in Science Teaching.

Dörnyei, Z. (2005). The psychology of the language learner: Individual differences in second Language acquisition. Mahwah, NJ: Lawrence Erlbaum.

Ferguson, J. M., \& Dorman, J. P. (2001). Psychosocial classroom environment and academic efficacy in Canadian high school mathematics classes. Alberta Journal of Educational Research, 47, 276-279.

Hanke, C. Y. C. (2013). A cross-national study of students' perceptions of mathematics classroom environment, attitudes towards mathematics and academic self-efficacy among middle school students in Hong Kong and the USA (Doctoral dissertation, Curtin University). Retrieved from https://espace.curtin.edu.au/bitstream/handle/20.500.11937/2575/199177_Hanke\%22014.pdf?sequence $=2$

Lim, C. T. D. (2013). Learning environments in English classrooms in Singapore: Determinants and effects (Doctoral dissertation). Retrieved from https://espace. curtin.edu.au/handle/20.500.11937/829

Madu, N. E. (2010). Associations between teachers' interpersonal behaviour, classroom learning environment and students' outcomes. Curtin University of Technology, Science and Mathematics Education Centre. Retrieved from http://espace.library.curtin.edu.au/R?func $=$ dbin-jump-full\&local_base $=$ gen 01 -era02\&object_id $=151667$

McMahon, S. D., Wernsman, J., \& Rose, D. S. (2009). The relation of classroom environment and school belonging to academic self-efficacy among urban fourth and fifth grade students. The Elementary School Journal, 109(3), 267-281.
Midgley, C., Maehr, M. L., Hruda, L. Z., Anderman, E., Anderman, L., Freeman, K. E., Gheen, M., Kaplan, A., Kumar, R., Middleton, M. J., Nelson, J., Roeser, R., \& Urdan, T. (2000). Manual for the patterns of adaptive learning scales. Ann Arbor, MI: University of Michigan.

Pajares, F. (1996). Self-efficacy beliefs in academic settings. Review of Educational Research, 66, 543-578.

Raoofi, S., Tan, B. H., \& Chen, S. H. (2012). Self-efficacy in second/foreign language learning contexts. English Language Teaching, 5(11), 60-73.

Spinner, H., \& Fraser, B. J. (2005). Evaluation of an innovative mathematics program interms of classroom environment, student attitudes, and conceptual development. International Journal of Science and Mathematics Education, 3(2), 267-293. doi:10.1007/s10763-0046531-8

Valle, A., Núñez, J. C., Cabanach, R. G., González-Pienda, J. A., Rodríguez, S., Rosário, P., \& Cerezo, R. (2009). Academic goals and learning quality in higher education students. The Spanish Journal of Psychology, 12(1), 96-105.

Wang, M. T. (2012). Educational and career interests in math: A longitudinal examination of the links between classroom environment, motivational beliefs, and interests. Developmental Psychology, 48(6), 1643-57. doi:10.1037/a0027247

Williams, M., \& Burden, R. L. (1997). Psychology for language teachers: A social constructivist approach. Cambridge: Cambridge University Press.

Zedan, R., \& Bitar, J. (2014). Environment learning as a predictor of mathematics self-efficacy and math achievement. American International Journal of Social Science, 3(6), 85-97.

Zimmerman, B. J. (1995). Self-efficacy and educational development. In A. Bandura (Ed.), Self-efficacy in changing societies (pp. 202-231). New York: Cambridge University Press.

Zimmerman, B. J., \& Kitsantas, A. (1997). Developmental phases in self-regulation: Shifting from process goals to outcome goals. Journal of Educational Psychology, 89(1), 29-36.

Zimmerman, B. J., \& Kitsantas, A. (2005). Homework practices and academic achievement: The mediating role of self-efficacy and perceived responsibility beliefs. Contemporary Educational Psychology, 30, 397-417.

Zimmerman, B. J., \& Kitsantas, A. (2007). Reliability and validity of the Self-Efficacy for Learning Form (SELF) scores of college students. Journal of Psychology, 215(3), 157-163. 